\title{
Evolution of a Bifunctional Reductase/Diels-Alderase for Fungal Indole Alkaloid Biosynthesis
}

Qingyun Dan ${ }^{a}$, Sean A. Newmister ${ }^{a}$, Kimberly R. Klas ${ }^{b}$, Amy E. Fraley ${ }^{a, c}$, Robert S. Paton ${ }^{b}$, K. N. Houk ${ }^{d}$, Robert M. Williams ${ }^{b}$, David H. Sherman ${ }^{\mathrm{a}, \mathrm{c}, \mathrm{e}}$ and Janet L. Smith ${ }^{\mathrm{a}, \mathrm{f}}$

aLife Sciences Institute, University of Michigan, Ann Arbor, Michigan 48109, USA, qingydan@umich.edu

${ }^{b}$ Department of Chemistry, Colorado State University, Fort Collins, Colorado 80523, USA

'Department of Medicinal Chemistry, University of Michigan, Ann Arbor, Michigan 48109, USA

${ }^{d}$ Department of Chemistry \& Biochemistry, University of California, Los Angeles, California, 90095, USA

${ }^{\mathrm{e}}$ Departments of Chemistry and Microbiology \& Immunology, University of Michigan, Ann Arbor, Michigan 48109, USA

fDepartment of Biological Chemistry, University of Michigan, Ann Arbor, Michigan 48109, USA, JanetSmith@umich.edu

Prenylated fungal indole alkaloids constituted of a bicyclo[2.2.2]diazaoctane core have attracted considerable interest due to their wide spectrum of biological activities [1], and thus serve as compelling targets for chemical synthesis and biosynthetic studies. Among them are the calmodulin-inhibitory malbrancheamides [2] and the anthelmintic paraherquamides [3,4] (Fig. 1a). We elucidated the malbrancheamide biosynthetic pathway (Fig. 1b) through complementary approaches [5] of biomimetic total syntheses to access the natural alkaloid in racemic form and in vitro enzymatic reconstitution to provide the natural antipode (+)-malbrancheamide. This demonstrated a common biogenetic scheme of reductive cleavage of a L-Pro-L-Trp dipeptide from a nonribosomal peptide synthetase (NRPS) followed by a cascade of reactions culminating in an intramolecular [4 + 2] hetero-Diels-Alder (IMDA) cyclization. Enzymatic synthesis of optically pure (+)-malbrancheamide requires MalC, a bifunctional NADPH-dependent reductase/Diels-Alderase with the ability to rescue oxidized products to cycloaddition. We elucidated the structural basis for IMDA catalysis with crystal structures of MalC $(1.6 \AA)$ and its homolog PhqE from the paraherquamide pathway $(2.1 \AA$, with substrate/product complex). The complementary structures are compelling examples of protein evolution in molecular detail, as both MalC and PhqE evolved from an ancestral short-chain dehydrogenase/reductase (SDR) to catalyze NADPH-dependent reduction coupled to diastereo- and enantioselective Diels-Alder cyclization. Our work reveals a new SDR subfamily with unexpected catalytic amino acids, a new group of Diels-Alder enzymes [6,7], and novel structural insights into the nature of IMDA catalysis [8]. The fungal indole alkaloid biosynthetic pathways also represent a novel "toolkit" for chemoenzymatic diversification of natural products with opportunities for facile access to improved therapeutics to treat human and animal diseases. The crystal structure determinations of MalC and PhqE had unique and complementary complications that will be highlighted in the presentation. 

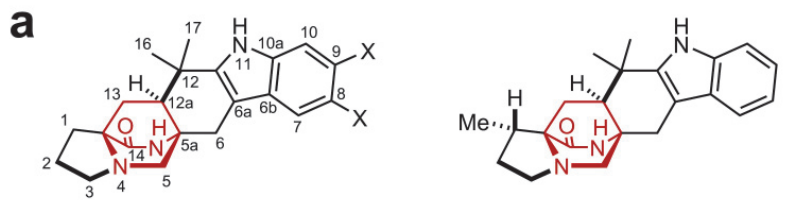

$(+)$-premalbrancheamide $(+)-1 \mathrm{X}=\mathrm{H} \quad(+)$-preparaherquamide $(+)-3$ $(+)$-malbrancheamide $(+)-2 \mathrm{X}=\mathrm{Cl}$

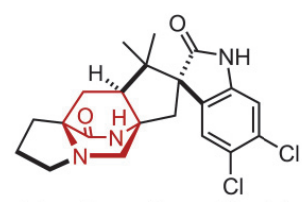

(-)-spiromalbramide (-)-4

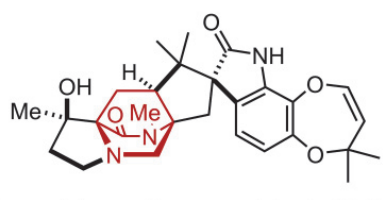

(-)-paraherquamide A (-)-5

b
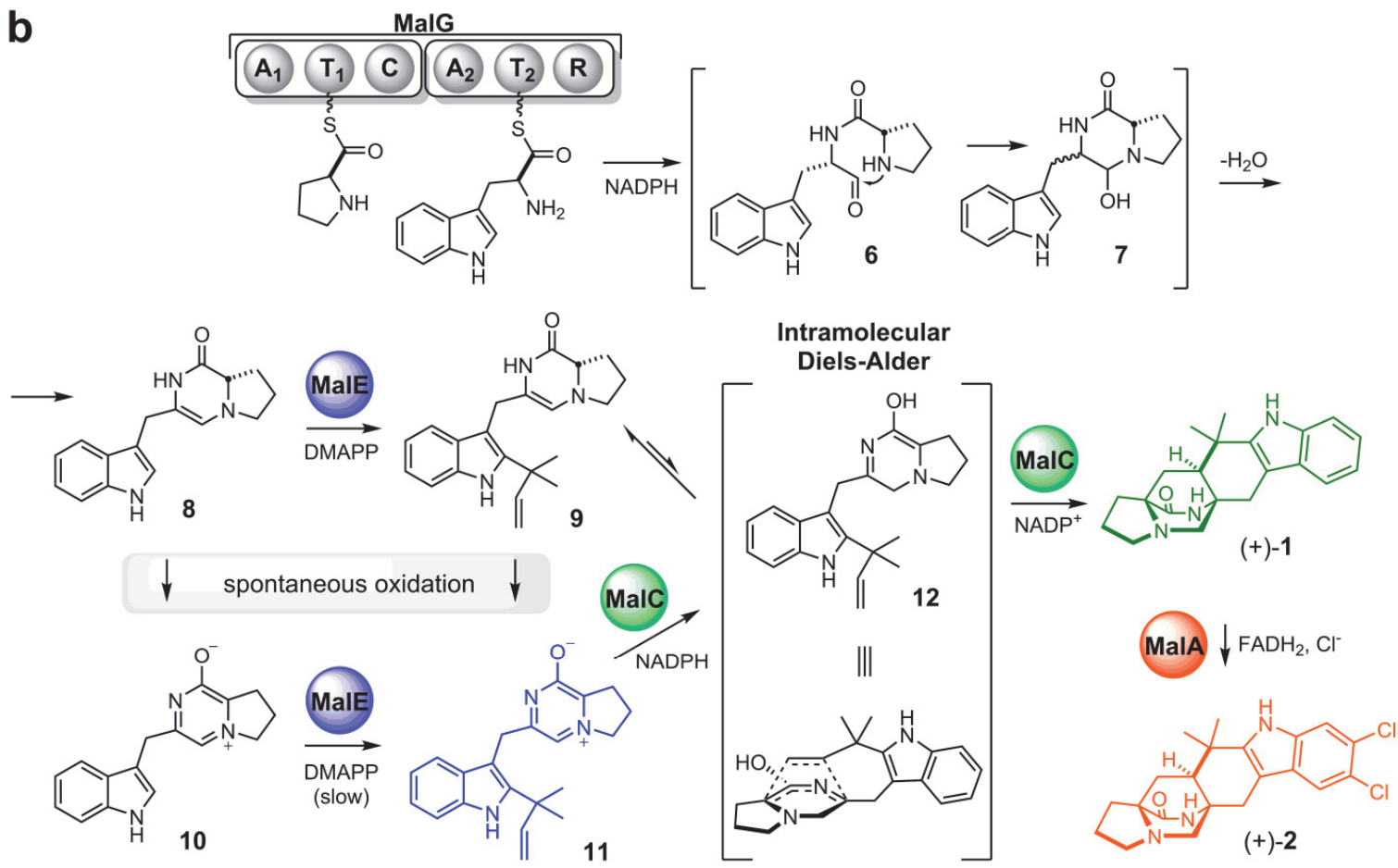

Figure 1. Fungal bicyclo[2.2.2]diazaoctane indole alkaloids and biosynthesis.

a. Representative natural products with the bicyclo[2.2.2]diazaoctane group colored in red. b. Scheme of malbrancheamide biosynthesis. The natural substrates are L-proline and L-tryptophan, and the final product is malbrancheamide (+)-2. The product of each biosynthetic step is colored differently. Proteins are indicated by spheres; MalG domains are adenylation $\left(A_{1}\right.$ and $\left.A_{2}\right)$, thiolation $\left(T_{1}\right.$ and $\left.T_{2}\right)$, condensation $(C)$ and reductase $(R)$.

\section{$\underline{\text { References }}$}

[1] Klas, K. R. et al. Nat Prod Rep 35, 532-558 (2018).

[2] Martinez-Luis, S. et al. Tetrahedron 62, 1817-1822 (2006).

[3] Robertson, A. P. et al. J Pharmacol Exp Ther 303, 853-860 (2002).

[4] Little, P. R. et al. Vet Parasitol 181, 180-193 (2011).

[5] Li, S. et al. MedChemComm 3, 987-996 (2012).

[6] Kim, H. J. et al. Nature 473, 109-112 (2011).

[7] Ohashi, M. et al. Nature 549, 502-506 (2017).

[8] Yang, Z. et al. Trends in Chemistry 1, 22-34 (2019). 